\title{
Benchmarking angular-momentum projected Hartree-Fock as an approximation
}

\author{
Stephanie M. Lauber \\ Hayden C. Frye \\ Calvin W. Johnson \\ E-mail: cjohnson@sdsu.edu \\ San Diego State University, 5500 Campanile Drive, San Diego, CA 92182-1233
}

\begin{abstract}
We benchmark angular-momentum projected-after-variation HartreeFock calculations as an approximation to full configuration-interaction results in a shell model basis. For such a simple approximation we find reasonably good agreement between excitation spectra, including for many odd- $A$ and odd-odd nuclides. We frequently find shape coexistence, in the form of multiple HartreeFock minima; mixing in shape coexistence, the first step beyond single-reference projected Hartree-Fock, demonstrably improves the spectrum in the $s d$ - and $p f$ shells. The complex spectra of germanium isotopes present a challenge: for even $A$ the spectra are only moderately good and those of odd $A$ bear little resemblance to the configuration-interaction results. Despite this failure we are able to broadly reproduce the odd-even staggering of ground state binding energies, save for germanium isotopes with $N>40$. To illustrate potential applications, we compute the spectrum of the recently measured dripline nuclide ${ }^{40} \mathrm{Mg}$. All in all, projected Hartree-Fock often provides a better description of low-lying nuclear spectra than one might expect. Key to this is the use of gradient descent and unrestricted shapes.
\end{abstract}

Submitted to: J. Phys. G: Nucl. Part. Phys. 


\section{Introduction}

The excitation spectra of atomic nuclei exhibit a variety of very different behaviors. The archetypal patterns of rotational, vibrational, and pairing (or seniority) are evident in even-even nuclides, and leave their fingerprints on the complex spectra of odd- $A$ and even odd-odd nuclides. In response, a variety of methods and approximations have been developed to model atomic nuclei [1].

Of these approaches, mean-field methods such as Hartree-Fock (HF) are the most common starting points, for their relative simplicity, appeal to intuition, and flexibility. Here one has independent particles or quasi-particles moving in a field generated by averaging over the rest of the system. In some cases, such as the interacting shell model [2, 3, 4, 5, 6, the mean-field fades away to the background: the many-body basis states are antisymmetrized products of single-particle states with good angular momentum. The interacting shell model, also known as configuration-interaction (CI) is flexible and can easily generate excited states, but requires a large basis to build up correlations. Hence the key to the interacting shell model is to have a simple, efficient representation of the basis allowing for fast calculation of matrix elements of the Hamiltonian [7].

Alternately, one can use fewer, more complex basis states, such as Green's function Monte Carlo [8] or coupled-cluster calculations [9. More closely related to CI are are beyond-mean-field methods such as generator-coordinate methods 11, 10, 11, 12, as well as the so-called Monte Carlo shell model [13, 14. These methods also expand the wave function in a basis, but while CI uses many simple states to build up correlations, generator coordinate methods and the Monte Carlo shell model uses fewer but more complex states with some correlations already built in. In particular, these methods construct mean-field states deformed by external fields, either based upon our physics understanding (quadrupole and pairing fields in generator coordinate methods) or sampled stochastically (Monte Carlo shell model). Here conserved symmetries, e.g. rotational invariance and, if using quasiparticles, particle number conservation, are broken and must be restored. Breaking and subsequently restoring symmetries builds in important correlations in the wave functions, as well as leading naturally to common excitation features such as rotational bands.

Thus at the heart of beyond-mean-field methods is the projection of broken symmetries [1, 15, for example angular-momentum projected Hartree-Fock calculations, and generalizations to quasiparticles, number and angular-momentum projected Hartree-Fock-Bogoliubov calculations. In this paper we will show that the excitation spectra coming out of simple angular-momentum projected-after-variation Hartree-Fock (PHF), when compared against full configuration-interaction (FCI) calculations, are surprisingly good, not only for strongly deformed even-even nuclides, but for a much wider variety of cases, including odd-A and odd-odd nuclides. We specifically allow for unrestricted deformation, and find the minima by gradient descent, an important ingredient. While pairing is known to be an important correlation in nuclei, motivating generalizations to quasiparticles and (projected) Hartree-Fock-Bogoliubov (HFB), we investigate neutron separation energies and especially the odd-even staggering (OES) of the ground state energy, and find our calculations systematically recover most, though not all, of the OES. In many cases we naturally find a second minimum, often referred to as shape coexistence [16]; we go beyond strict single-reference PHF and mix in shape coexistence states, which can improve the excitation spectra. As a particular challenge we consider the complex 
spectra of germanium isotopes. We conclude with a calculation of ${ }^{40} \mathrm{Mg}$, near the neutron dripline and recently measured experimentally [17, and tentatively identify one of the experimentally measured states as a $0^{+}$produced by a second minimum. Thus, although going to either FCI or, when impossible, generator coordinate or Monte Carlo shell model methods are often important, the very starting point, PHF is already a very good one for describing nuclear excitation spectra. PHF is also used in quantum chemistry [15], another motivation for our benchmarking.

\section{Methods}

In order to compare the projected Hartree-Fock calculations against full configuration interaction results, we carry out our calculations in the same shell-model space using the same interaction input.

We have four different spaces with interactions adjusted for each one: the $s d$ shell, comprising a frozen ${ }^{16} \mathrm{O}$ core with valence $1 s_{1 / 2}-0 d_{3 / 2}-0 d_{5 / 2}$ orbitals, with the universal $s d$ interaction version $B$ (USDB) [18; the $p f$ shell, comprising a frozen ${ }^{40} \mathrm{Ca}$ core with valence $1 p_{1 / 2}-1 p_{3 / 2}-0 f_{5 / 2}-0 f_{7 / 2}$ orbitals, with a modified $G$-matrix interaction (GX1A) interaction [19, 20, 21]; for our germanium calculations we assumed a ${ }^{56} \mathrm{Ni}$ core and active valence space $1 p_{1 / 2}-1 p_{3 / 3}-0 f_{5 / 2}-0 g_{9 / 2}$, and use the JUN45 interaction [22]. Finally, for our calculation of ${ }^{40} \mathrm{Mg}$ we worked in the $s d-p f$ space without restrictions, with an ${ }^{16} \mathrm{O}$ core, and used a monopole-modified universal interaction [23].

\subsection{Configuration-interaction}

We benchmark against full configuration-interaction (FCI), sometimes called the interacting shell model [2, 3, 4, 5, 6], where one expands the wave function in a many-body basis $\{|\alpha\rangle\}$ :

$$
|\Psi\rangle=\sum_{\alpha} c_{\alpha}|\alpha\rangle .
$$

For our basis we use antisymmetrized products of single-particle states, or Slater determinants: if $\hat{a}_{i}^{\dagger}$ is the creation operator for the $i$ th single-particle state, then the occupation representation of an $A$-body Slater determinant is

$$
\hat{a}_{1}^{\dagger} \hat{a}_{2}^{\dagger} \hat{a}_{3}^{\dagger} \ldots \hat{a}_{A}^{\dagger}|0\rangle
$$

where $|0\rangle$ is the fermionic vacuum, or, equivalently, a frozen core.

Because both total angular momentum $\hat{J}^{2}$ and the $z$-component $\hat{J}_{z}$ commute with our Hamiltonians, we choose many-body basis states with fixed eigenvalues of the latter, labeled as $M$. This is known as an $M$-scheme basis, and is easily accomplished when using single-particle states $i$ with good angular momentum $j_{i}$ and $z$-component $m_{i}$, so that the total value of $J_{z}$ of $(2)$ is $m_{1}+m_{2}+m_{3}+\ldots m_{A}$. Other than fixing $J_{z}$, for FCI we take all possible Slater determinants. In this framework it is easy to construct an orthonormal many-body basis, $\langle\alpha \mid \beta\rangle=\delta_{\alpha \beta}$. The code we use [7, 24] efficiently computes matrix elements of the Hamiltonian in this basis, $\langle\alpha|\hat{H}| \beta\rangle$, and then uses the Lanczos algorithm to find low-lying eigenstates [6, 25]. It is these FCI results that we use as a benchmark, against which we compare PHF. 


\subsection{Hartree-Fock and finding minima}

Our Hartree-Fock code (unpublished but described in [26]) works in a shell-model space, that is, the single particle states are expanded in an occupation basis: we minimize $\langle\hat{H}\rangle$ for an arbitrary Slater determinant $|\Psi\rangle$. In particular, we redefine the single-particle basis by an $N_{s} \times N_{s}$ unitary transformation, where $N_{s}$ is the number of single-particle states,

$$
\hat{c}_{a}^{\dagger}=\sum_{i} U_{i a} \hat{a}_{i}^{\dagger},
$$

(the only restriction we impose is $U_{i a}$ is real; this is equivalent to an $R T$ symmetry, that is, the wave function is invariant under a rotation by $\pi$ about the $y$-axis, followed by time reversal $T$ ), and then let

$$
|\Psi\rangle=\hat{c}_{1}^{\dagger} \hat{c}_{2}^{\dagger} \ldots \hat{c}_{A}^{\dagger}|0\rangle \text {. }
$$

Hence the Slater determinant is represented as a rectangular matrix $\Psi$, which for $N_{p}$ particles is given by $N_{p}$ columns, each of length $N_{s}$, of the matrix $\mathbf{U}$ in Eq. (3). We use separate proton and neutron Slater determinants. One can compute $\langle\hat{H}\rangle$ for any $\Psi$ and then vary the elements of $\mathbf{U}$ to minimize [26].

One can define local minima by using Thouless' theorem [1] to define an energy landscape in Slater determinants. Because we compare two methods of finding minima, and inquire if these states are indeed minima, we review here the relevant details. Given any reference Slater determinant $|\Psi\rangle$, we separate single-particle states into occupied states labeled by $i, j$ and unoccupied states labeled by $m, n$. One can then define a particle-hole excitation relative to the reference state, $\hat{c}_{m}^{\dagger} \hat{c}_{i}|\Psi\rangle \equiv\left|m i^{-1}\right\rangle$. The condition for a extremum, and thus a necessary condition for a minimum, is the Hartree-Fock condition:

$$
\left\langle\Psi|\hat{H}| m i^{-1}\right\rangle=\left\langle\Psi\left|\left[\hat{H}, \hat{c}_{m}^{\dagger} \hat{c}_{i}\right]\right| \Psi\right\rangle=0 .
$$

Often one introduces the one-body Hartree-Fock effective Hamiltonian, $h_{a b}=$

$\left\langle\Psi\left|\left[\hat{H}, \hat{c}_{a}^{\dagger} \hat{c}_{b}\right]\right| \Psi\right\rangle$, where $a, b$ can be any of $m, i$, and then the Hartree-Fock condition is simply stated as $h_{m i}=0$. An equivalent condition is

$$
[\mathbf{h}, \rho]=0,
$$

where $\rho_{a b}=\left\langle\Psi\left|\hat{c}_{a}^{\dagger} \hat{c}_{b}\right| \Psi\right\rangle$ is the density matrix, with $\rho=\Psi \boldsymbol{\Psi}^{\dagger} ; \boldsymbol{\Psi}$ is the matrix representation of the Slater determinant.

If $\mathbf{h}$ is diagonal, then the off-diagonal terms $h_{m i}$ must vanish. Thus, a typical strategy is to diagonalize $h_{a b}$, and use the lowest eigenvectors of $\mathbf{h}$ to construct the matrix $\boldsymbol{\Psi}$. However $\mathbf{h}$ depends upon $|\Psi\rangle$ and hence diagonalization must be carried out self-consistently. We iterated diagonalization until we reached convergence in energy; this, however, is not necessary converged in the Slater determinant and is not guaranteed to find an energy minimum: see, for example, in Ref. [1] the discussion accompanying Fig. 5.3 how diagonalization can oscillate between two solutions, a situation we often encountered with odd numbers of particles. Nonetheless, iterated diagonalization is widely used, even in publicly-available and widely used codes [27.

To check whether or not one is at an extremum, we check the Hartree-Fock condition (6) is satisfied. To check whether one is at a local minima, we can find the eigenvalues of the stability matrix [1],

$$
S=\left(\begin{array}{cc}
\mathbf{A} & \mathbf{B} \\
\mathbf{B}^{*} & \mathbf{A}^{*}
\end{array}\right)
$$


where $A_{m i, n j}=\left\langle m i^{-1}|\hat{A}| n j^{-1}\right\rangle$ and $B_{m n, i j}=\left\langle m n i^{-1} j^{-1}|\hat{H}| \Psi\right\rangle$. The stability matrix is related to the famous matrix formulation of the random phase approximation (RPA), with a positive-definite stability matrix guaranteeing real RPA frequencies. As a simpler test, $\mathbf{A}$ is the matrix form of the Tamm-Dancoff approximation (TDA), whose eigenstates are one-particle, one-hole approximations of excited states [1, 3]; the eigenvalues of the TDA must also be positive definite for a minimum.

We refer to true local minima, that is, self-consistent solutions that satisfy all of our criteria (the Hartree-Fock condition (6) and non-negative eigenvalues for the stability matrix-zero eigenvalues arise from degeneracy under rotation) as stable minima. In particular we call the stable global minimum the $H F$ minimum, or the primary minimum, and other stable local minima as secondary minima. While it is not unreasonable to think of secondary minima as examples of shape coexistence [16], it is possible to find states with the same "shape" (i.e. quadrupole deformation parameters $\beta$ and $\gamma$, which we find by diagonalizing the mass quadrupole tensor as in (28), but which are demonstrably different states, for example that they have significantly different expectation values of spin or pairing. Conversely, with a pure pairing interaction we find multiple stable minima with the exact same HF energy but with dramatically different shape parameters and even different expectation values of angular momentum $J^{2}$.

Solutions from diagonalization that are converged in energy but which nonetheless fail one or more of our tests for a true minimum (satisfying the Hartree-Fock condition (6), or either of the TDA or stability matrix having negative eigenvalues) we call unstable solutions. In section 3.1 we discuss the stability of solutions found selfconsistently through diagonalization: diagonalization often but not always found stable minima for even-even nuclides, but failed to do so for odd-A and odd-odd nuclides. Modifications such as taking linear combinations of old and new $\mathbf{h}$ did not appreciably change this scenario.

Often one calls upon various measures for odd numbers of particles, such as blocking [27, 29] or the equal filling approximation [30. Instead we turn to an alternative to diagonalization: gradient descent, 1, 31, which more reliably finds stable minima. Here one explores an energy surface by applying

$$
\left|\Psi^{\prime}\right\rangle=\exp \left(-\lambda \sum_{m i} Z_{m i} \hat{c}_{m}^{\dagger} \hat{c}_{i}\right)|\Psi\rangle
$$

where we let $Z_{m i}=h_{m i}$. The minima we find are generally stable (true) minima, and always had the same energy or lower than solutions found by diagonalization. While secondary stable local minima are common, we only found a small number of saddlepoints, that is, solutions which satisfy the Hartree-Fock condition (6) but for which the stability matrix has at least one negative eigenvalue. For example, in the $s d$ shell with the USDB interaction, ${ }^{26} \mathrm{Ne}$ has a true prolate minimum at $-77.26 \mathrm{MeV}$ with $\beta=0.12$, but also has an oblate saddlepoint at $-76.26 \mathrm{MeV}$ and $\beta=0.07$.

As discussed below, the quality of the spectra, i.e., agreement with FCI, was generally as good or better with gradient descent minima. In particular, for odd numbers of particles (odd-odd and odd-A), the energies of Slater determinants found by gradient descent were almost always significantly lower than those found by diagonalization. Although gradient descent takes more time to converge to a solution than diagonalization, it was nonetheless sufficiently fast we did not need sophisticated optimization. Because with either diagonalization or gradient descent we can rapidly generate many solutions, we generate a large number, typically 10-20, from random 
starting points; in our experience this seems to be sufficient to find all self-consistent solutions. Increasing the number of random starting points to 1000 did not yield additional solutions. Convergence of gradient descent is sped up by doing 10 or 20 pre-diagonalizations. Because of its consistently superior performance, we strongly recommend gradient descent as the preferred method of finding minima.

\subsection{Projected Hartree-Fock}

Aside from having good particle number, we do not impose any constraints, such as axial symmetry, on our Slater determinants (save, for historic reasons, that the matrix representation of the Slater determinant $\boldsymbol{\Psi}$ be real). Allowing arbitrary deformation is important: in general, if one has two minima, one with axial symmetry and one without, the triaxial deformed state is usually lower in energy. As a further example of the importance of triaxiality, previous work on proton-neutron random phase approximation (RPA) of Gamow-Teller transitions [32, found that triaxial HF reference states better reproduced the FCI transition strengths than the reference states with axial symmetry; furthermore, RPA using triaxially deformed Hartree-Fock reference states did a better job than quasi-particle RPA using spherical HartreeFock-Bogoliubov reference states, at least in the $p f$ shell. Hence allowing arbitrary deformations is a desirable ingredient for our work here.

An arbitrary state $|\Psi\rangle$, including Slater determinants, can be expanded as sum of states with good angular momentum quantum number $J, K$, where $J$ is the total angular momentum and $K$ the $z$-component in the intrinsic frame:

$$
|\Psi\rangle=\sum_{J, K} c_{J, K}|J K\rangle
$$

Let $\hat{P}_{M K}^{J}$ be a projection operator that projects out a state of good angular momentum $J$ and $z$-component $K$ in the intrinsic frame, but rotated to $z$ component $M$. In the standard approach one accomplishes this by an integral [1, but we perform the projection by solving a set of linear algebra equations [33, 34]. Then one computes the angular-momentum projected Hamiltonian and overlap kernels, respectively:

$$
\begin{aligned}
H_{M K}^{J} & \equiv\left\langle\Psi\left|\hat{H} \hat{P}_{M K}^{J}\right| \Psi\right\rangle, \\
N_{M K}^{J} & \equiv\left\langle\Psi\left|\hat{P}_{M K}^{J}\right| \Psi\right\rangle,
\end{aligned}
$$

and solves the generalized eigenvalue problem,

$$
\sum_{K} H_{M K}^{J} g_{K}^{(r)}=E \sum_{K} N_{M K}^{J} g_{K}^{(r)} .
$$

where $r$ labels the eigenpairs for a given angular momentum $J$. Note that we are doing projection after variation, rather than the computationally more intensive variation after projection. These matrices are all of small dimension, and in our model spaces, the HF calculations takes a few seconds and the PHF under a minute on a modest laptop.

\section{Results}

Here we present our results. Note that ours are not the first tests of PHF against full CI shell model calculations [35, 36, 37] (there have also been similar benchmarks of PHFB [38]). This study is, however, the widest systematic benchmarking of PHF 


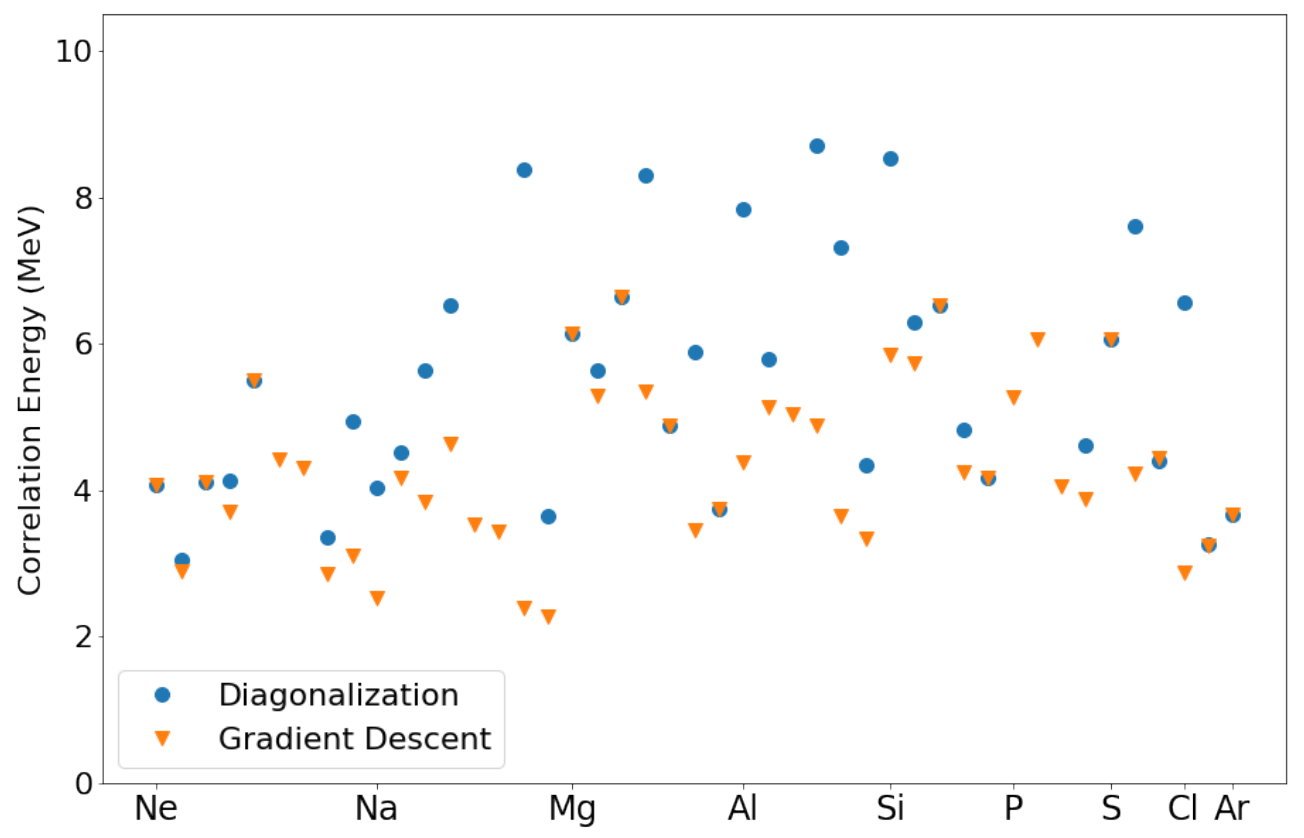

Figure 1. Comparison of finding Hartree-Fock solutions by diagonalization and gradient descent. Here we plot the Hartree-Fock correlation energy (the difference between the HF energy and the FCI ground state energy) for $s d$-shell nuclides, using the USDB interaction [18. In all cases where the diagonalization correlation energies are higher, the HF self-consistent solutions are not actual minima.

against FCI, emphasizing a variety of different nuclides including odd-A and odd-odd nuclides. Furthermore, while we obviously cannot reproduce the FCI binding energies with PHF, in section 3.6 we look at systematics of the odd-even staggering of the binding energy, which is expected to be sensitive to pairing.

\subsection{Diagonalization versus gradient descent}

An important question is the relative efficacy of diagonalization and gradient descent finding Hartree-Fock minima. Fig. 1 compares the HF correlation energies (that is the energy difference between the unprojected Hartree-Fock solution and the FCI ground state energy) for self-consistent solutions found by diagonalization and gradient descent, sample all $s d$-shell nuclides with $N \geq Z$ and $2 \leq Z \leq 10\left({ }^{20} \mathrm{Ne}-{ }^{36} \mathrm{Ar}\right)$. Not shown are 8 outliers found by diagonalization with $\mathrm{HF}$ correlation energies greater than $10 \mathrm{MeV}:{ }^{25,26} \mathrm{Ne},{ }^{26,27} \mathrm{Na},{ }^{28} \mathrm{Al}$, and ${ }^{30,31,32} \mathrm{P}$.

In all even-even $s d$-shell nuclides save ${ }^{26} \mathrm{Ne}$, diagonalization and gradient descent found equivalent HF minima, that is, the same except for randomly different orientations. (Orientations are found by taking the expectation values of components of angular momentum, $\left\langle\hat{J}_{x}^{2}\right\rangle$, etc..) Diagonalization found only unstable solutions for ${ }^{26} \mathrm{Ne}$, about $6 \mathrm{MeV}$ higher in energy than the true stable minima, a case we have found no explanation for. In all odd-A and odd-odd nuclides, however, gradient descent found a lower, stable minimum, and diagonalization only found unstable solutions; and all of the unstable solutions found by diagonalization, save for ${ }^{26} \mathrm{Ne}$, are odd-A or 


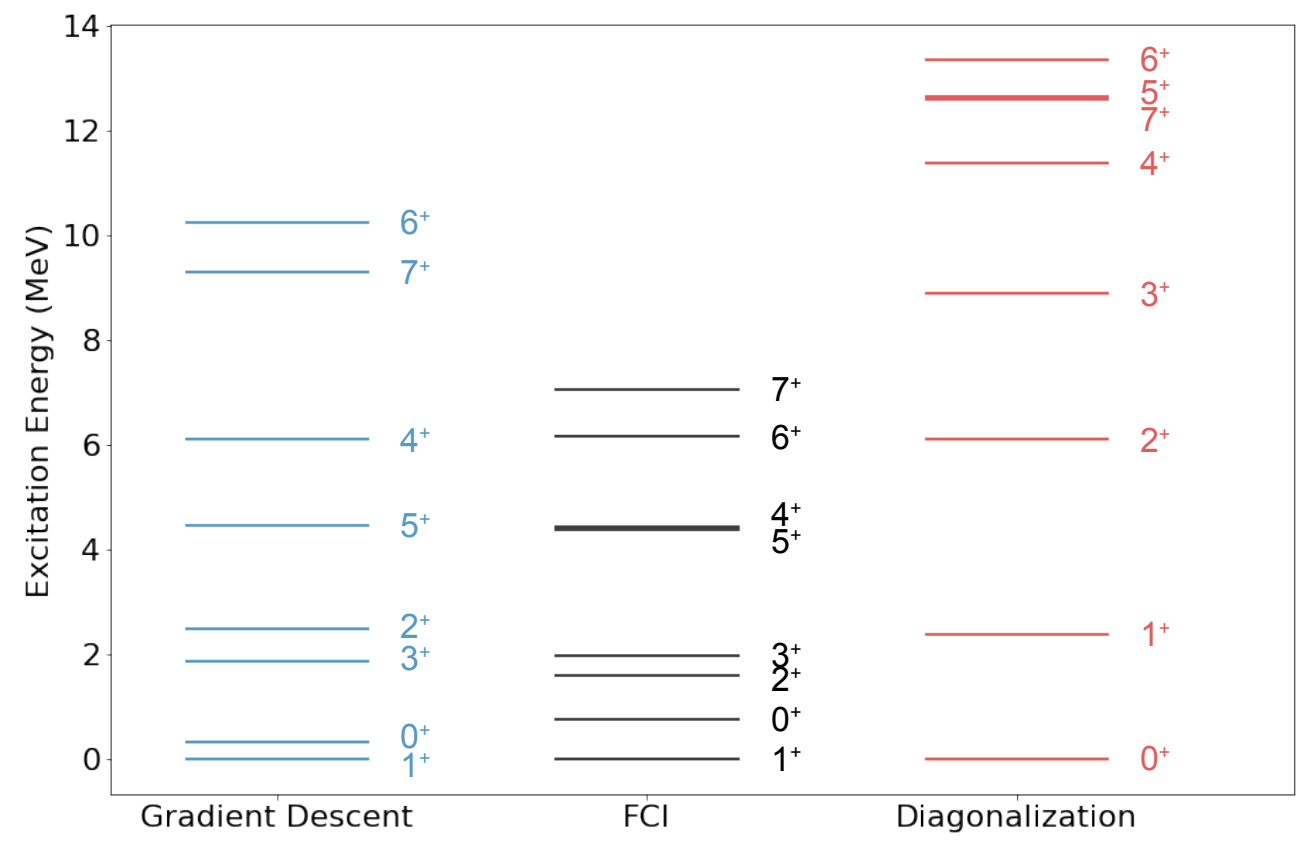

Figure 2. Excitation spectra of ${ }^{30} \mathrm{P}$. In the middle is the benchmark excitation spectrum from full configuration interaction (FCI), while to the left and and right are PHF excitation spectra based upon Hartree-Fock solutions found by gradient descent and diagonalization, respectively.

odd-odd nuclides. Conversely, all solutions found by gradient descent satisfy the HF condition Eq. (6), and aside from a very small number of saddle points all were stable. For all $s d$-shell nuclides, the average difference in energy between the (true, stable) HF minimum found by gradient descent, and solutions, stable or unstable found by diagonalization is $\sim 4 \mathrm{MeV}$.

We found a similar result for the $p f$ shell: in only a handful of even-even cases (5 out of 45, taking $22 \leq Z \leq N \leq 38$ ) did diagonalization not find the same global minimum as gradient descent, and for those five cases the selfconsistent diagonalization solution was unstable; while in only five odd- $A$ nuclides did diagonalization find the same global minimum as gradient descent. In no odd-odd cases did diagonalization find the same stable global minimum as gradient descent.

In addition to lowering the HF energies, gradient descent minima provide better PHF excitation spectra than solutions from diagonalization. As an example, we provide the excitation spectra for ${ }^{30} \mathrm{P}$ in Fig. 2. This is a somewhat extreme case: for diagonalization, the HF energy is $-122.141 \mathrm{MeV}$ and the PHF g.s. energy is -126.493 $\mathrm{MeV}$, while for the gradient descent minimum the HF and PHF g.s. energies are $-148.912 \mathrm{MeV}$, and $-151.188 \mathrm{MeV}$, respectively.

In the $s d$-shell, gradient descent found stable secondary minima, interpretable as shape coexistence, in about $40 \%$ of the cases. (Diagonalization found far more secondary self-consistent solutions, but these often did not satisfy the Hartree-Fock condition Eq. (6) and/or were unstable; for the rest of this paper, we will focus exclusively on solutions found through gradient descent.) In most cases the excitation spectrum was qualitatively improved by the addition of a second (local) minimum. 


\begin{tabular}{|c|r|c|r|r|c|r|}
\hline Nucleus & $\begin{array}{r}E_{\mathrm{HF}, 1} \\
(\mathrm{MeV})\end{array}$ & $\beta_{1}$ & $\begin{array}{r}\gamma_{1} \\
(\mathrm{deg})\end{array}$ & $\begin{array}{r}E_{\mathrm{HF}, 2} \\
(\mathrm{MeV})\end{array}$ & $\beta_{2}$ & $\begin{array}{r}\gamma_{2} \\
(\mathrm{deg})\end{array}$ \\
\hline${ }^{20} \mathrm{Ne}$ & -36.404 & 0.45 & 0 & -31.831 & 0.23 & 60 \\
\hline${ }^{25} \mathrm{Mg}$ & -89.113 & 0.23 & 19 & -89.089 & 0.23 & 19 \\
\hline${ }^{29} \mathrm{Na}$ & -104.784 & 0.09 & 0 & -104.692 & 0.09 & 0 \\
\hline${ }^{30} \mathrm{Si}$ & -148.238 & 0.12 & 47 & -148.097 & 0.11 & 60 \\
\hline${ }^{30} \mathrm{Al}$ & -137.851 & 0.10 & 34 & -137.592 & 0.10 & 28 \\
\hline${ }^{34} \mathrm{Cl}$ & -199.663 & 0.06 & 60 & -198.554 & 0.06 & 60 \\
\hline${ }^{46} \mathrm{Ti}$ & -67.306 & 0.32 & 0 & -66.474 & 0.24 & 59 \\
\hline${ }^{48} \mathrm{~V}$ & -92.037 & 0.26 & 0 & -91.851 & 0.26 & 0 \\
\hline${ }^{62} \mathrm{Ni}$ & -261.307 & 0.12 & 60 & -260.473 & 0.08 & 1 \\
\hline${ }^{63} \mathrm{Zn}$ & -282.779 & 0.11 & 0 & -282.020 & 0.10 & 60 \\
\hline${ }^{64} \mathrm{Cu}$ & -285.099 & 0.10 & 53 & -284.709 & 0.07 & 1 \\
\hline${ }^{64} \mathrm{Ni}$ & -277.062 & 0.07 & 44 & -276.767 & 0.05 & 0 \\
\hline
\end{tabular}

Table 1. Hartree-Fock energies, $\beta$ and $\gamma$ deformation parameters for first 2 stable minima for the excitation spectra in Fig. 4 and 5

Additionally, mixing in a second minimum lowered the ground state energy. The amount of lowering varied strongly: in many cases it was only a few tens of keV, while ${ }^{30} \mathrm{Si}$ was lowered by $0.63 \mathrm{MeV},{ }^{46} \mathrm{Ti}$ by $0.44 \mathrm{Mev},{ }^{70} \mathrm{Ge}$ by $0.73 \mathrm{MeV}$ and ${ }^{71} \mathrm{Ge}$ by 1.17 $\mathrm{MeV}$. For the 21 cases plotted in this paper, the average lowering of the ground state energy of nuclides was $0.22 \mathrm{MeV}$. In the $s d$ - and $p f$-shell the average lowering of the ground state energy was 0.16 and $0.18 \mathrm{MeV}$, respectively, while the germanium ground states were lowered by about $0.3 \mathrm{MeV}$. The shape parameters $\beta, \gamma$ for the plotted $s d$ and $p f$-shell nuclides are found in Table 1, while those for germanium isotopes are in Table 2,

In many cases PHF, while a crude approximation, nonetheless reproduces excitation spectra, not only for rotational spectra as one might naively expect, but in general far better than we anticipated. Naturally adding a second minimum often improves the spectra. In this paper we strove to present a general and balanced representation of results, not only the cases where PHF and especially the inclusion of a second minimum yields a good result, but also less successful cases.

In the next subsection we quantify the goodness of PHF spectra compared to the FCI benchmark spectra. We then present example excitation spectra from $s d$-shell nuclides, while in section 3.4 we present examples from the $p f$-shell. Germanium isotopes are known to have triaxial shapes and complex excitation spectra, with even$A$ Ge isotopes typically displaying multiple low-lying $0^{+}$states from shape coexistence [16, 39, 40, and so we investigate them as a challenging case in section 3.5 .

Although clearly one would expect the PHF ground state energy to be a poor estimate of the absolute binding energy, we nonetheless investigate in section 3.6 the relative binding energies through neutron separation energies and odd-even staggering of binding energies. Here one might expect PHF to fail, as the HF solutions lack pairing correlations. We note, however, that odd-even staggering has been demonstrated as robust in generic random two-body interactions, even when explicit $J=0$ two-body matrix elements, i.e., those associated with pairing, are eliminated [1]. Furthermore, HFB calculations in this region using the same shell-model interactions exhibit only 
weak pairing, especially for well-deformed nuclei

Once again the results are surprisingly better than we expected. Only when we get to germanium isotopes with $N>40$ do we see a significant deviation, perhaps signalling the increased role of pairing.

\subsection{Goodness of excitation spectra}

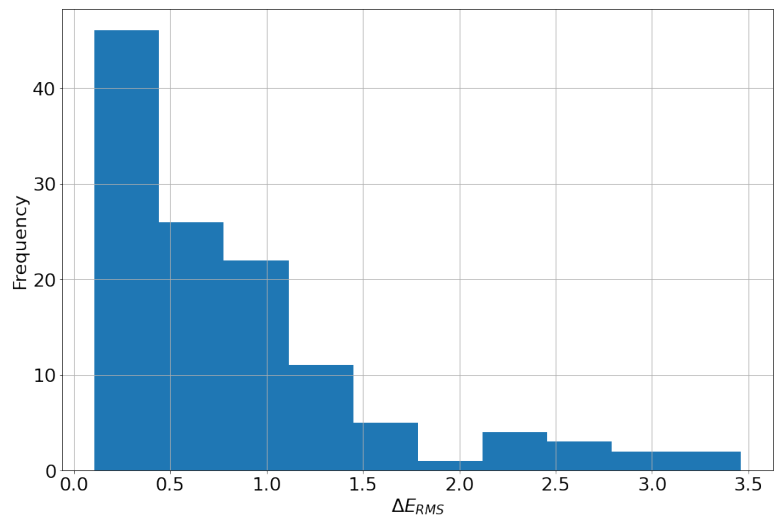

Figure 3. The root-mean-square error in the PHF excitation spectra relative to FCI spectra, Eq. 13, calculated for 114 nuclei across the $s d$ - and $p f$-shells and 8 even-even germanium isotopes. The average error was $0.85 \mathrm{MeV}$ for the total set and $0.38 \mathrm{MeV}$ for germanium.

To approximately quantify the goodness of the PHF excitation spectra, we computed the root-mean-square error for $N_{\text {lev }}$ levels in the excitation spectrum of a given nuclide,

$$
\Delta E_{\mathrm{RMS}}^{2}=\frac{1}{N_{\text {lev }}} \sum_{\alpha=1}^{N_{\text {lev }}}\left(E_{\alpha}^{\mathrm{FCI}}-E_{\alpha}^{\mathrm{PHF}}-E_{\text {shift }}\right)^{2} .
$$

Here we matched FCI and PHF levels with the same $J$, in order of their appearance. $E_{\text {shift }}$ is a floating shift that allows for different ground state angular momenta or other aspects: for example, in many cases the spacing in PHF between a $0^{+}$ground state and the first $2^{+}$state is significantly smaller than for FCI, while other levels have the approximately correct spacing. We found $E_{\text {shift }}$ by minimizing $\Delta E$. Because we could not always be certain we correctly matched physically corresponding levels, if the separation between a PHF and matched FCI level was greater than $2 \Delta E_{\mathrm{RMS}}$ we excluded that pair and recomputed. While this is far from a rigorous procedure, in nonetheless provides quantitative insight into the quality of the PHF spectra. In our figures below we have the PHF spectra shifted by $E_{\text {shift }}$.

Fig. 3 shows the distribution of $\Delta E_{\mathrm{RMS}}$ for 114 nuclides across the $s d$ - and $p f$ shell and 8 even- $A$ germanium isotopes with more than 2 matched pairs of PHF and FCI energies. The average error was $0.85 \mathrm{MeV}$. For even-even nuclides the average error was $0.67 \mathrm{MeV}$, for odd-odd nuclides 0.68 , and for odd- $A 1.05 \mathrm{MeV}$; all had a Poisson-like distribution much as Fig. 3 For the germanium isotopes the average error was lower than the total set at $0.38 \mathrm{MeV}$; this smaller number is likely due to their

$\ddagger$ C.-F. Jiao, private communication 
compressed energy spectra and the presence of a second HF minima in 7 out of the 8 nuclei investigated. For comparison, the rms error between empirical FCI calculations and experimental spectra is typically on the order of $0.15-0.30 \mathrm{MeV}[5]$.

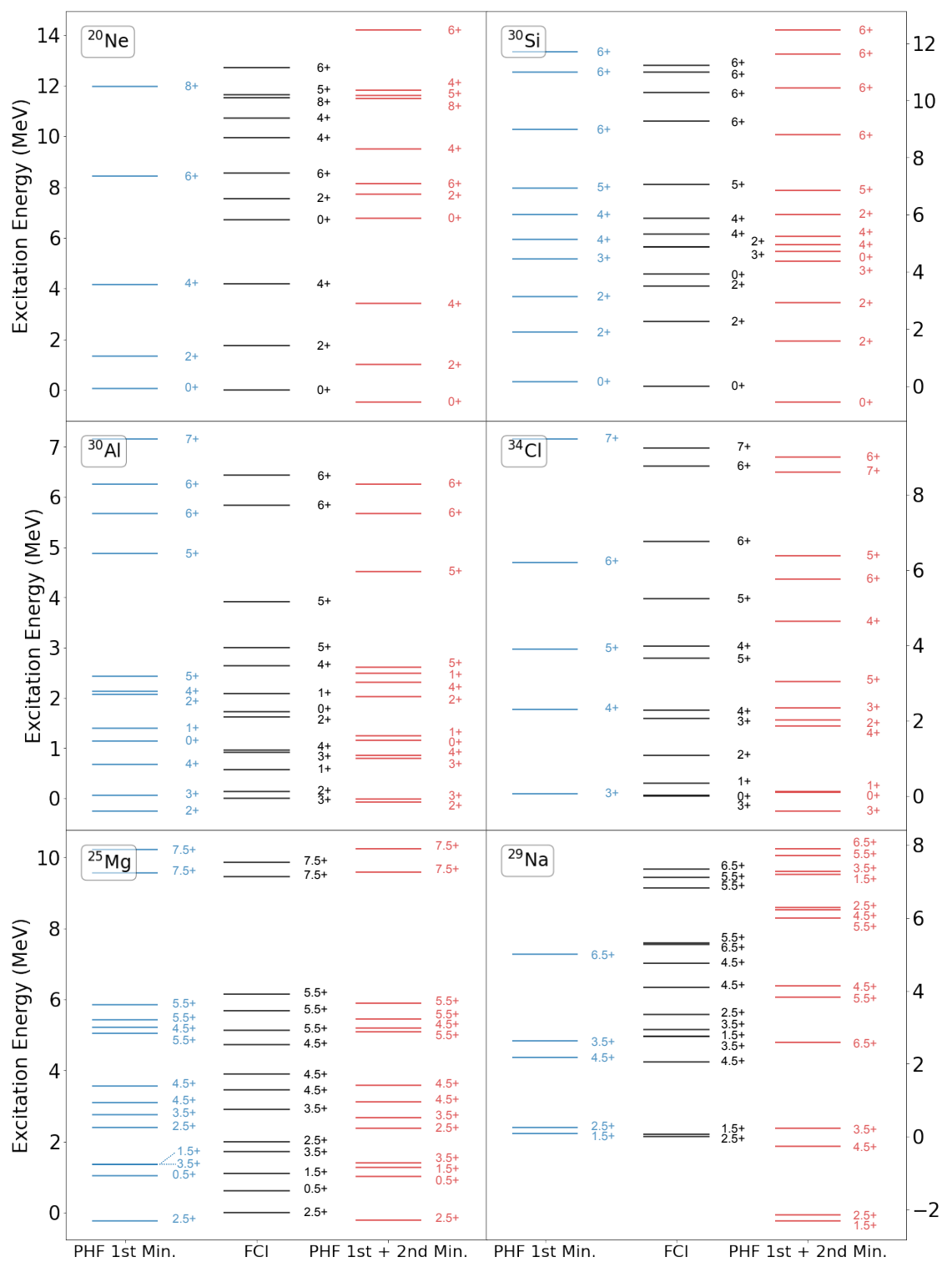

Figure 4. Excitation spectra for selected $s d$-shell nuclides, using the USDB interaction 18, computed in FCI (middle spectra), in PHF with the primary global HF minimum (left-hand spectra), and PHF mixing the primary and secondary HF minima (right-hand spectra). 


\subsection{Examples from the sd-shell}

We chose six illustrative cases in the $s d$-shell, shown in Figure 4 two even-even nuclei $\left({ }^{20} \mathrm{Ne}\right.$ and $\left.{ }^{30} \mathrm{Si}\right)$, two odd-odd nuclei $\left({ }^{30} \mathrm{Al}\right.$ and $\left.{ }^{34} \mathrm{Cl}\right)$ and two odd-A nuclei $\left({ }^{25} \mathrm{Mg}\right.$ and ${ }^{29} \mathrm{Na}$ ). All had stable primary and secondary minima with different Hartree-Fock energies and deformation parameters. As we allowed the PHF excitation spectra to float in order to miminize $\Delta E_{\mathrm{RMS}}$, the ground states do not completely align. In many cases this allows us to see that the PHF spectra tend to be less compressed than the FCI spectra, a phenomenon found not only in the $s d$-shell but also in the $p f$-shell.

Even-even. In both even-even nuclei, the second minimum reproduces a second low lying $0^{+}$state, a second bandhead, at roughly the correct excitation energy. In ${ }^{20} \mathrm{Ne}$, the second miminum roughly reproduces a second rotational band. In ${ }^{30} \mathrm{Si}$, the $0_{2}^{+}$state is correctly placed between the $2_{2}^{+}$and $3_{1}^{+}$state found in FCI calculations.

Odd-odd. PHF fails to provide the correct $3^{+}$ground state for ${ }^{30} \mathrm{Al}$ but the addition of the second minimum lowers the $3_{1}^{+}$excitation energy by roughly $800 \mathrm{keV}$, nearly degenerate with the $2_{1}^{+}$state. Adding the second minimum also puts the $4_{1}^{+}$ and $3_{2}^{+}$very close in energy, as in the FCI spectrum.

A more significant improvement is found in ${ }^{34} \mathrm{Cl}$. While even $\mathrm{PHF}$ with a single minimum produces the correct $3^{+}$ground state, including the second minimum produces the $0_{1}^{+}, 1_{1}^{+}$states just above the ground state, as well as the $3_{2}^{+}$at near the correct excitation energy.

Odd- $A$. The addition of the second minimum for ${ }^{25} \mathrm{Mg}$ lowered the ground state by only $1 \mathrm{keV}$, the smallest change in the $s d$-shell nuclei in this study. Here PHF, while roughly reproducing the ordering of low-lying states, clusters them in a way not reflected in the FCI spectrum, and addition of the second minimum only mixes minimally. Conversely, while PHF fails (barely) to get the correct ground state spin for ${ }^{29} \mathrm{Na}$, even the the HF global minimum alone reproduces the tight clustering of the $5 / 2_{1}^{+}, 3 / 2_{1}^{+}$states and of the $7 / 2_{1}^{+}, 9 / 2_{1}^{+}$, while also placing the $11 / 2_{1}^{+}$and $13 / 2_{1}^{+}$ at roughly the right place.

\subsection{Examples from the $p f$-shell}

The 6 benchmark cases for the $p f$-shell are shown in Figure 5 with three even-even nuclei $\left({ }^{46} \mathrm{Ti}\right.$ and $\left.{ }^{62,64} \mathrm{Ni}\right)$, two odd-odd nuclei $\left({ }^{48} \mathrm{~V}\right.$ and $\left.{ }^{64} \mathrm{Cu}\right)$ and one odd-A nucleus $\left({ }^{63} \mathrm{Zn}\right)$. Similar to the $s d$-shell results (above) we see improvement in the excitation spectra for the even-even nuclei, modest improvement in the odd-odd nuclei and the case where the addition of a second local minimum produces energy level doublets similar to those seen in FCI.

Even-even. As expected, the even-even nuclei show improvement in their spectra with the addition of the second minimum correctly reproducing a second $0^{+}$state for both. In ${ }^{46} \mathrm{Ti}$, the excitation spectrum from just the prolate global $\mathrm{HF}$ minimum provides a mediocre approximation to the exact FCI results, but the inclusion of the second minimum adds $2_{2}^{+}$and $5_{1}^{+}$, in reversed order but in approximately the right places, with the same for the $3_{1}^{+}, 0_{2}^{+}, 8_{1}^{+}$states. Similar behavior occurs for ${ }^{62} \mathrm{Ni}$, which has an approximate vibrational spectrum: the spectrum from the oblate global $\mathrm{HF}$ minimum alone is closer to rotational, but mixing in the second local minimum dramatically improves this, with the cluster of $0_{2}^{+}, 2_{2}^{+}, 4_{1}^{+}$in roughly the right place, albeit with the $0_{2}^{+}$and $4_{1}^{+}$reversed.

Conversely, the excitation spectrum of ${ }^{64} \mathrm{Ni}$ from just the triaxial global $\mathrm{HF}$ 


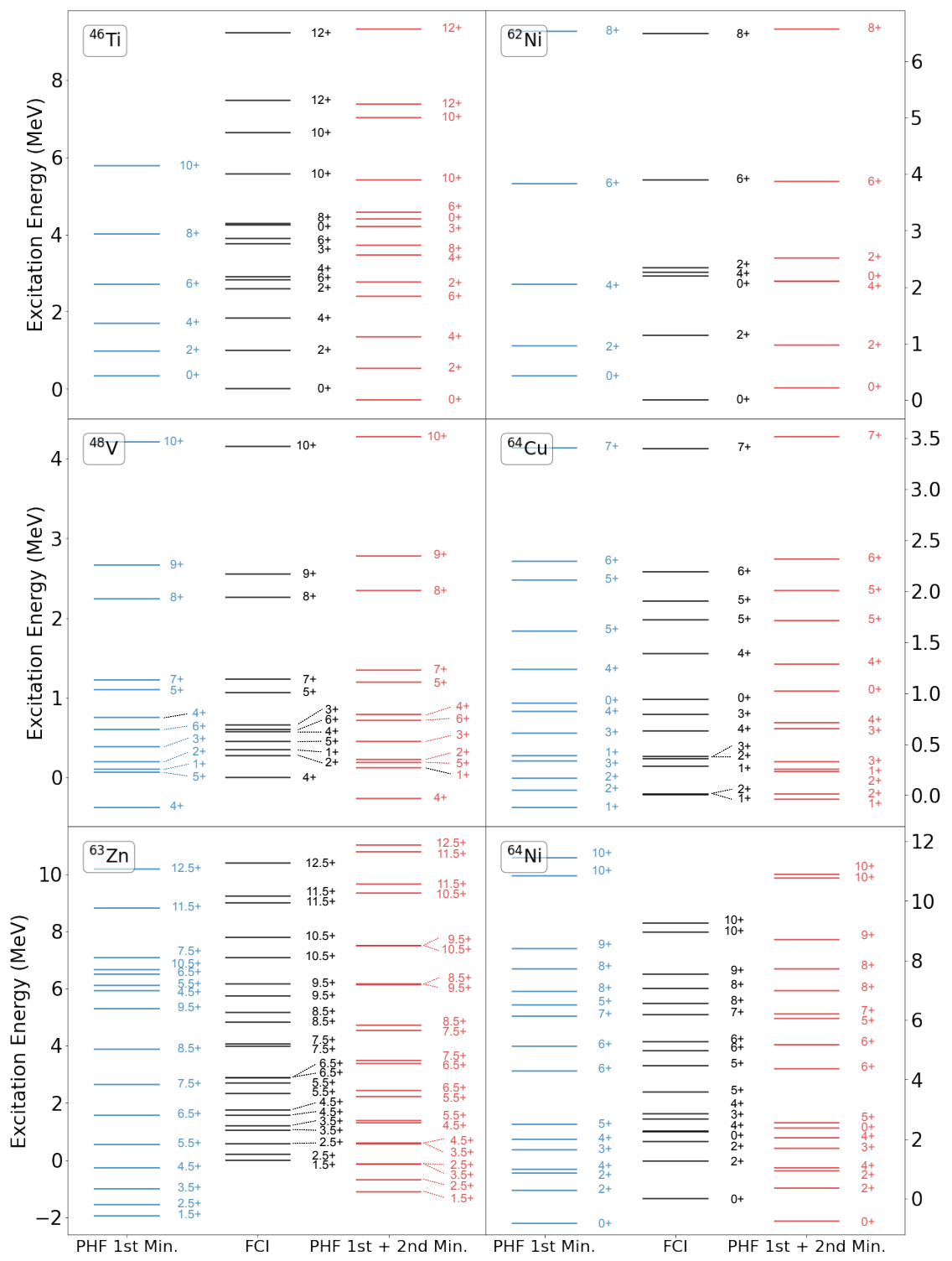

Figure 5. Same as Fig. 4 but for selected $p f$-shell nuclides using the GX1A interaction 19, 20, 21].

minimum is already reasonably good, getting the ordering of the first several states and their spacing approximately correct, save for the $0_{2}^{+}$state which only appears with the addition of a second local minimum.

One trend to note here is the $2_{1}^{+}$excitation energy tends to be systematically low, more so in the $p f$-shell than in the $s d$-shell. In section 3.5 we will see this trend in germanium isotopes as well. One possible explanation is that the $0^{+}$ground state is in fact not low enough, due to inadequate treatment of pairing. As we will discuss in our conclusions, however, the evidence regarding pairing is mixed. 
Odd-odd. Even with just the global HF minimum, the low-lying PHF excitation spectrum ${ }^{48} \mathrm{~V}$ matches qualitatively the FCI spectrum, getting the correct ground state spin and the low-lying states in approximately the correct order, in particular the neardoublets of $5_{3}^{+}, 7_{1}^{+}$and $8_{1}^{+}, 9_{1}^{+}$, as well as the approximate location of the $10_{1}^{+}$. Addition of a second local minimum only slightly changes the spectrum. Conversely, ${ }^{64} \mathrm{Cu}$ is significantly improved by the addition of the second HF minimum, reproducing the ground state near-doublet $1_{1}^{+}, 2_{1}^{+}$and the $1_{2}^{+}, 2_{2}^{+}, 3_{1}^{+}$cluster around $300 \mathrm{kev}$. The $0_{1}^{+}$ state is placed correctly near $1 \mathrm{MeV}$ excitation energy. This is all the most impressive when noting the high density of low-lying states.

$O d d-A$. The FCI spectrum of ${ }^{63} \mathrm{Zn}$ is characterized by $5 / 2^{+}, 7 / 2^{+}, 9 / 2^{+}, 11 / 2^{+}$ doublets. When projecting from only the prolate HF global minimum, we get only one of each doublet, at approximately the correct excitation energy; adding the second, oblate local minimum produces the angular momentum partners but not in the correct relative positions.

\subsection{Shape coexistence: Ge isotopes}

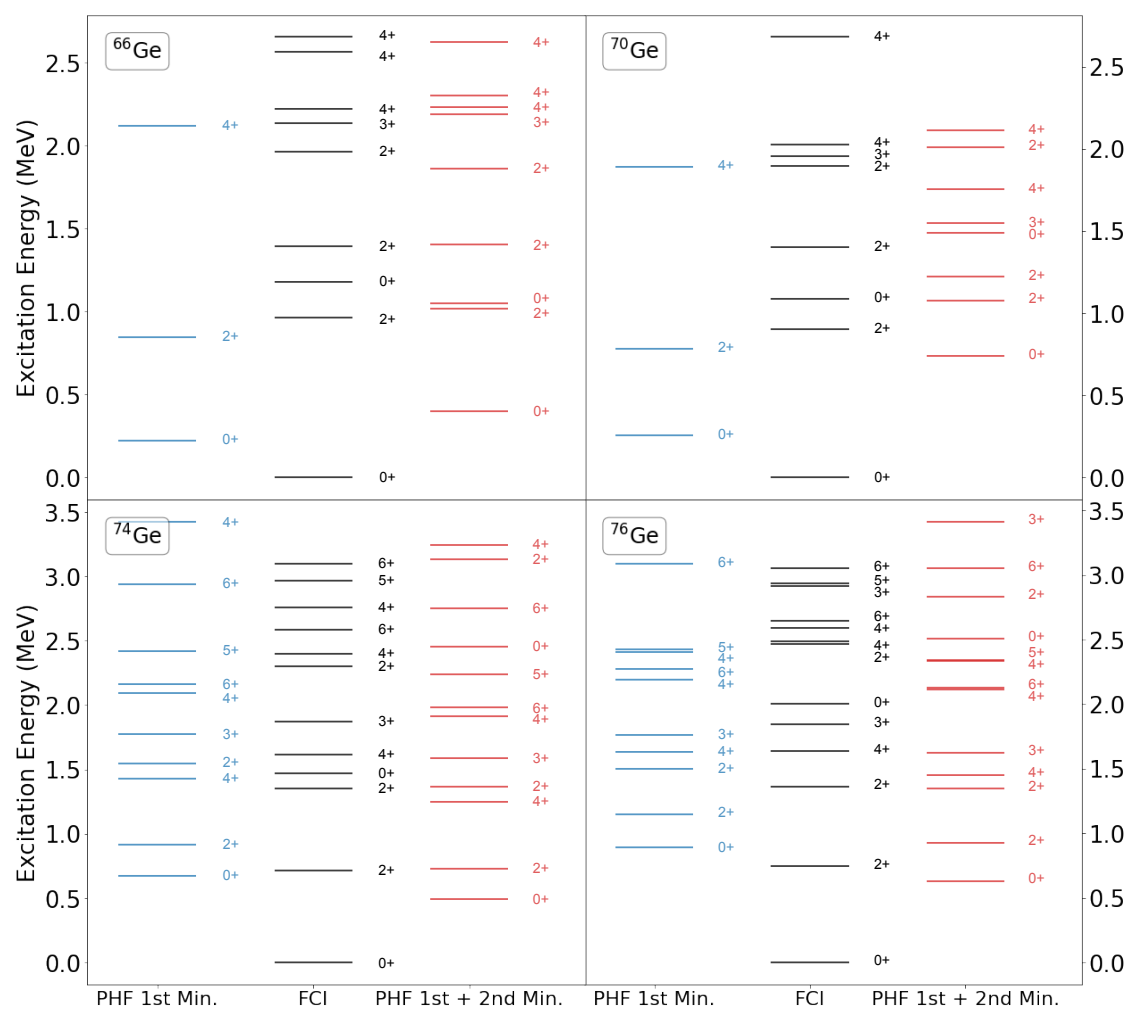

Figure 6. Same as Fig. 4 but for selected even- $A$ germanium isotopes. All calculations were performed using the JUN45 interaction [22], in the $f_{5 / 2} p g_{9 / 2}$ model space.

Germanium nuclei, known for their complex spectra arising from triaxiality and shape coexistence [39, 40, offer a strong test of PHF in capturing excitation spectra. 


\begin{tabular}{|c|c|cc|cc|c|}
\hline Nuclide & Force & $\beta_{1}$ & $\begin{array}{c}\gamma_{1} \\
(\mathrm{deg})\end{array}$ & $\beta_{2}$ & $\begin{array}{c}\gamma_{2} \\
(\mathrm{deg})\end{array}$ & $\begin{array}{c}\Delta E_{\text {soln }} \\
(\mathrm{MeV})\end{array}$ \\
\hline${ }^{64} \mathrm{Ge}$ & JUN45 & 0.28 & 20 & - & - & - \\
& JJ44 & 0.30 & 19 & 0.18 & 60 & 0.36 \\
& SLy6 & 0.24 & 28 & - & - & - \\
\hline${ }^{66} \mathrm{Ge}$ & JUN45 & 0.21 & 60 & 0.24 & 8.1 & 0.58 \\
& JJ44 & 0.25 & 12 & 0.21 & 58 & 0.17 \\
& SLy6 & 0.23 & 60 & 0.21 & 0.0 & 0.53 \\
\hline${ }^{68} \mathrm{Ge}$ & JUN45 & 0.17 & 38 & - & - & - \\
& JJ44 & 0.24 & 40 & 0.13 & 60 & 0.33 \\
& SLy6 & 0.21 & 39 & - & - & - \\
\hline${ }^{70} \mathrm{Ge}$ & JUN45 & 0.11 & 60 & 0.21 & 36 & 0.08 \\
& JJ44 & 0.21 & 34 & - & - & - \\
& SLy6 & 0.23 & 34 & 0.17 & 60 & 1.93 \\
\hline${ }^{72} \mathrm{Ge}$ & JUN45 & 0 & - & 0.16 & 44 & 0.87 \\
& JJ44 & 0.19 & 31 & 0.02 & soft & 3.35 \\
& SLy6 & 0.0 & - & 0.22 & 34 & 0.70 \\
\hline${ }^{74} \mathrm{Ge}$ & JUN45 & 0.15 & 41 & 0.06 & 60 & 1.35 \\
& JJ44 & 0.17 & 18 & 0.15 & 37 & 0.45 \\
& SLy6 & 0.24 & 28 & 0.07 & 0 & 4.90 \\
\hline${ }^{76} \mathrm{Ge}$ & JUN45 & 0.13 & 29 & 0.10 & 29 & 1.23 \\
& JJ44 & 0.13 & 23 & - & - & - \\
& SLy6 & 0.16 & 0 & 0.24 & 24 & -2.94 \\
\hline${ }^{78} \mathrm{Ge}$ & JUN45 & 0.09 & 12 & 0.10 & 21 & 0.74 \\
& JJ44 & 0.11 & 25 & - & - & - \\
& SLy6 & 0.18 & 0 & - & - & - \\
\hline
\end{tabular}

Table 2. Comparison of mean-field deformation parameters for even-even germanium isotopes, using two shell-model interactions in the $1 p_{1 / 2,3 / 2^{-}} 0 f_{5 / 2^{-}}$ $0 g_{9 / 2}$ space with a ${ }^{56} \mathrm{Ni}$ core, JUN45 22, and JJ44 [42, 43, as well as SkyrmeBCS calculations using the SLy6 parameterization [44] as tabulated in [40. Here $\beta_{1}, \gamma_{1}$ and $\beta_{2}, \gamma_{2}$ refer to the quadrupole shape parameters of the first and, if it exists, second HF minima, respectively. $\Delta E_{\text {soln }}$ is the energy difference between the first and second HF minima if the latter exists. 'Soft' here means a range of values of $\gamma$ had nearly identical energies, within the numerical tolerance of our code.

Table 2 gives the quadrupole shape deformation parameters for two shell-model interactions for the $1 p-0 f_{5 / 2}-0 g_{9 / 2}$ space, JUN45 [22, and a modified [43] version of the JJ44 interaction [42. These agree qualitatively well with density functional calculations [40, in particular the SLy6 parameterization 44] of Skyrme Hartree-Fock calculations plus BCS pairing of a zero-range pairing force [45]. Here $\beta_{1}, \gamma_{1}$ refer to the shape parameters of the global Hartree-Fock, or first, minimum, while $\beta_{2}, \gamma_{2}$ are the parameters for a secondary, shape coexistence minimum if it exists. $\Delta E_{\text {soln }}$ refers to the difference in Hartree-Fock energies between the global minimum and the shapecoexistence solution. (Note that for the SLy6 calculation of ${ }^{76} \mathrm{Ge}$, the order of the solutions is switched due to pairing, not included here.) In several cases different forces have similar solutions but in different order, e.g., for ${ }^{70} \mathrm{Ge}$, the global $\mathrm{HF}$ minimum for JUN45 is oblate with a triaxial shape coexistence solution, while for JJ44 and SLy6 the global minimum is triaxial and SLy6 has an oblate shape coexistence solution. 
While the agreement is not exact, the overall congruence nonetheless is noteworthy. For the even-even germanium isotopes, $\Delta E_{\mathrm{RMS}}=0.38 \mathrm{MeV}$; note that the the spectra in Fig. 6 is compressed compared to our $s d$ - and $p f$-shell examples.

As with the $s d$ - and $p f$-shells, Fig. 6 presents several excitation spectra of even- $A$ germanium isotopes, using the JUN45 interaction. Although the model space allows for unnatural parity states, our HF solutions for even- $A$ only had natural parity, and so we do not discuss the unnatural parity states in the spectra. Like our $p f$-shell examples, the $2_{1}^{+}$from projection is systematically too low in excitation energy, or, arguably, the $0_{1}^{+}$ground state is not low enough. This latter interpretation is bolstered by allowing $E_{\text {shift }}$ in Eq. (13) to float when minimizing $\Delta E_{\mathrm{RMS}}$, leading to the PHF ground state appearing $0.5 \mathrm{MeV}$ or more above the FCI ground state.

Both ${ }^{66,70} \mathrm{Ge}$ have oblate global HF minima with simple rotational excitation spectra; the mixing of the second, triaxial local minima in each case puts the $0_{2}^{+}$and other features in roughly the right place, albeit lower than in FCI. The ${ }^{66} \mathrm{Ge}$ PHF spectrum is better than that of ${ }^{70} \mathrm{Ge}$.

The spectra from the triaxial global HF minima only for ${ }^{74,76} \mathrm{Ge}$ are more complex, and mixing in the second local HF minima do little more than put a second $0^{+}$at approximately the right excitation energy; overall the agreement between PHF and FCI is poorer.

Even with the inclusion of stable shape coexistence states, the PHF results for odd $A$ germanium isotopes did not reflect the complex excitation spectra, and so we do not present them.

Overall, even with the inclusion of stable shape coexistence states, PHF excitation spectra do not perform as well in approximating the FCI spectra of germanium isotopes. One can turn this around: germanium is a stringent test of any beyondmean-field methodology.

\subsection{Separation energies and odd-even staggering}

As PHF provides a relatively simple ground state wave function, one cannot expect it to reproduce the same ground state binding energy as FCI. Hence our focus so far on excitation energies. Nonetheless, one can also inquire about relative binding energies. Here we consider the neutron separation energies, $S_{n}=B E(Z, N)-B E(Z, N-1)$, and the neutron odd-even staggering (OES) [46, 47,

$$
\Delta_{o}^{(3)}(N)=\frac{1}{2}[B E(Z, N+1)+B E(Z, N-1)-2 B E(Z, N)]
$$

where $B E(Z, N)$ is the binding energy for a given $Z, N$. Both the neutron separation energy and the odd-even staggering is expected to be sensitive to pairing, and could be tests of how the number-conserving PHF approximation may fail. On the other hand, odd-even staggering occurs in random interactions even with the explicit pairing matrix elements set to zero [4], so such an interpretation is not ironclad.

A comparison of the neutron separation energy of the PHF and FCI results show good agreement between the data sets in the $s d$-shell. Figure 7 shows the distributions of the PHF and FCI results as well as the distribution of the differences.

The $s d$-shell OES for PHF and FCI is shown in Figure 8 with the difference between the two methods shown on the same scale in the bottom panel. The average of the magnitude for the OES for PHF and FCI are $1.483 \mathrm{MeV}$ and $1.672 \mathrm{MeV}$, respectively, with the average magnitude of the difference only $0.378 \mathrm{MeV}$. We found 


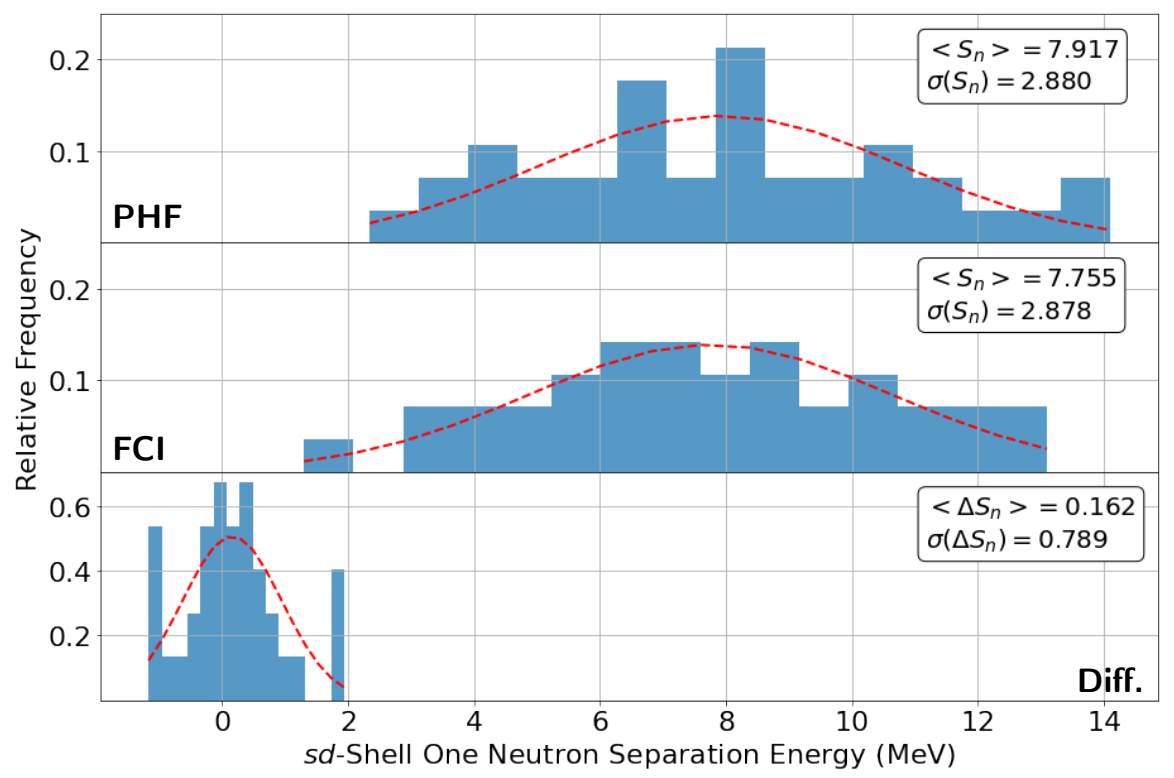

Figure 7. Distribution of neutron separation energies in the $s d$-shell computed with FCI and PHF, and the difference between the two methods.

that computing the OES with unprojected Hartree-Fock energies produced very poor results. We also found no strong evidence for shape staggering [46].

We also computed the OES for chromium and iron isotopes in the $p f$-shell, Fig. 9 , and for germanium isotopes, in Fig. 10. The former does very well. Interestingly, for the latter, we obtain reasonable agreement for $A<72$, with the PHF OES about two-thirds of the FCI values, but for $A \geq 72$, the PHF values are about a third of the FCI results. As this transition occurs at $N=40$ as one enters the $0 g_{9 / 2}$ shell, one plausible explanation is the lack of good treatment of pairing.

To test the role of pairing, we recomputed the OES for the chromium isotopic chain with increased pairing. Specifically, we added $-0.5 \hat{P}^{\dagger} \hat{P}$, where $\hat{P}^{\dagger}$ is the standard pair-creation operator, $\hat{P}^{\dagger}=\sum_{j, m>0} \hat{a}_{j, m}^{\dagger} \hat{a}_{j,-m}^{\dagger}$. This roughly doubles the strength of the $J=0, T=1$ matrix elements found in GX1A. The deformations were unchanged, but the PHF OES was roughly half that of the FCI OES. This provides further evidence that weak pairing in these nuclides allows a good reproduction of differences in binding energies. In heavier nuclei where pairing becomes more important, PHF may not work as well; but there it is more challenging to carry out systematic FCI calculations for benchmarking.

\subsection{Comparison to experimental ${ }^{40} \mathrm{Mg}$ results}

Finally, to demonstrate the utility of simple PHF calculations in a shell model basis, we consider the neutron-rich nucleus ${ }^{40} \mathrm{Mg}$, recently measured [48, 17. A calculation in the $s d-p f$ shell using a monopole-modified universal interaction [23, would require an $M$-scheme bais dimension of $10^{11}$, an order of magnitude larger than currently attainable. We compare the experimental results against PHF calculations in Fig. 11. We produce the first excited $2^{+}$state at roughly the right location, albeit a little low, 


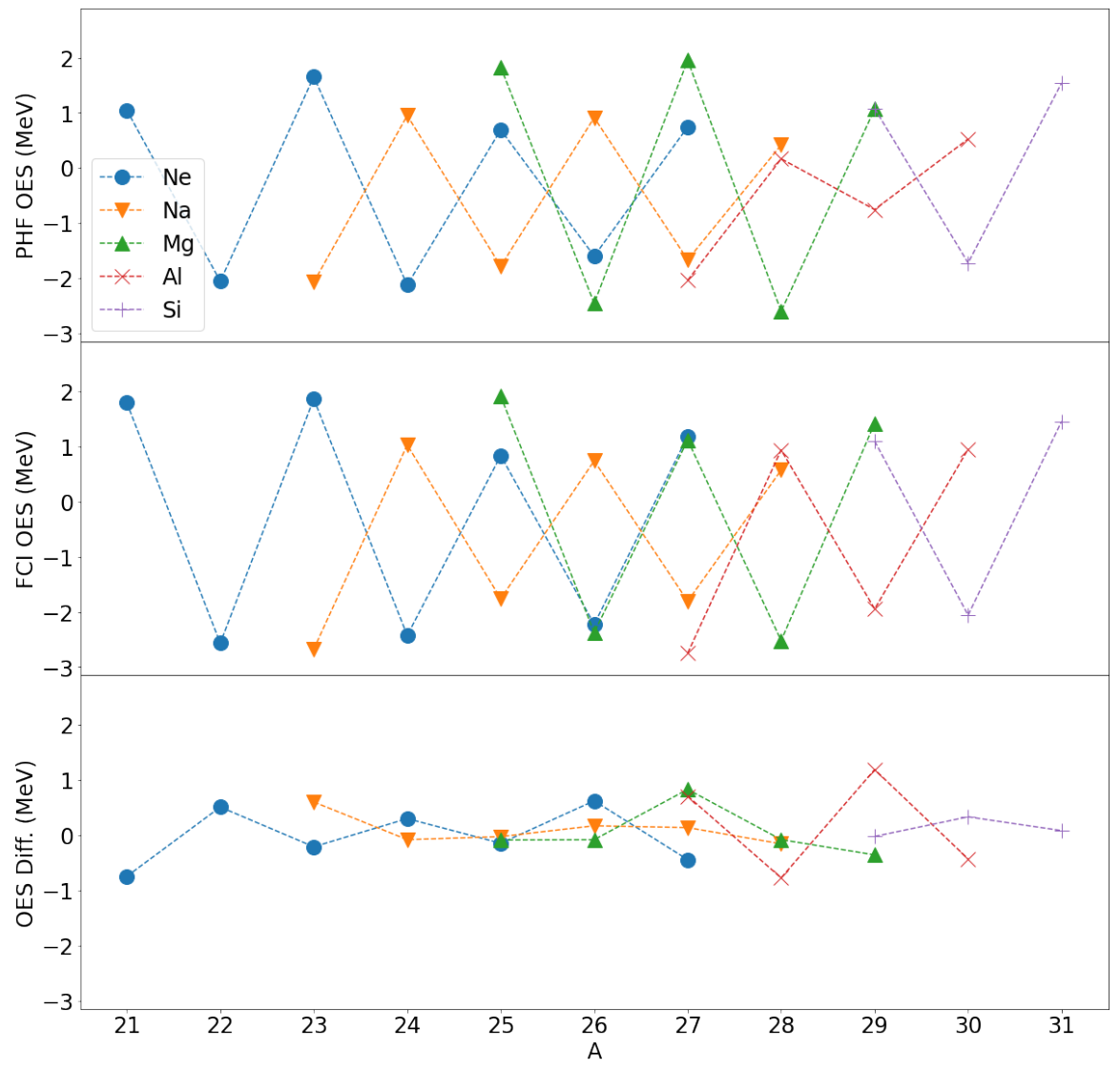

Figure 8. Odd-even staggering (OES) in $s d$-shell nuclei.

consistent with our other calculations. The $J^{\pi}$ of the second excited state has not been determined, but in our calculation it seems likely the $0_{2}^{+}$is the head of a second band arising from a second minimum.

\section{Conclusions and acknowledgements}

We have compared angular-momentum projected-after-variation Hartree-Fock spectra to full configuration-interaction calculations, carrying out both in the same shell-model framework, using the same interaction matrix elements. PHF, also in use in quantum chemistry 15, is a first step towards more sophisticated approximations, such as (projected) Hartree-Fock-Bogoliubov, generator coordinate, and the Monte Carlo shell model. Yet already, for a relatively simple and fast approximation PHF often provides qualitative agreement with FCI excitation spectra; key to this agreement are general deformations, including triaxiality, and the use of gradient descent to find the HF minima. Mixing in a second local minima when it exists, an additional step towards the more complex methods, often significantly improves the spectra. For our calculations the rms error in the spectra is around $0.78 \mathrm{MeV}$, with odd- $A$ performing significantly worse (rms error of about $1 \mathrm{MeV}$ ) than for even-even and odd-odd (rms error around 


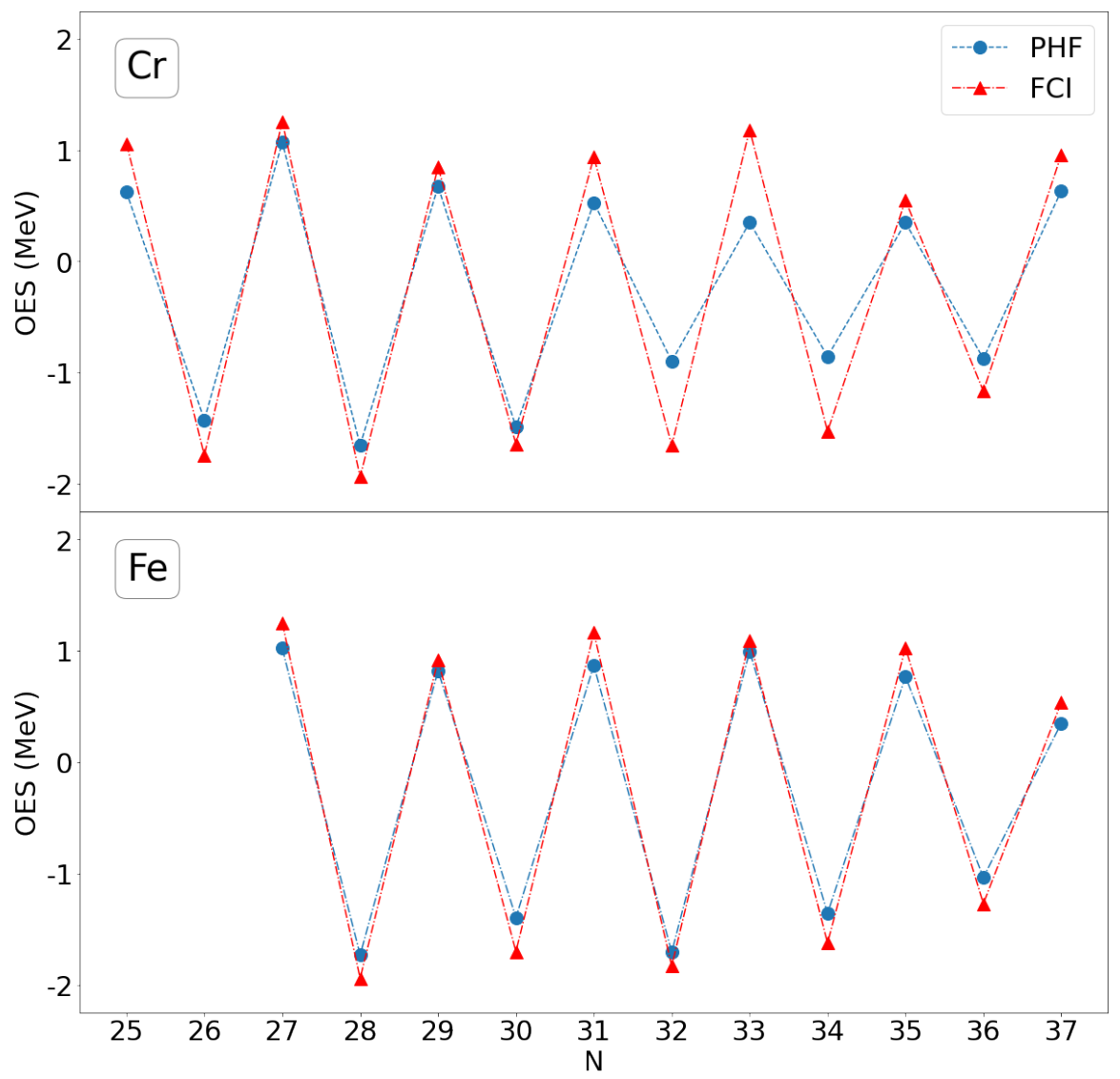

Figure 9. Odd-even staggering (OES) along the chromium (upper panel) and iron (lower panel) isotopic chains.

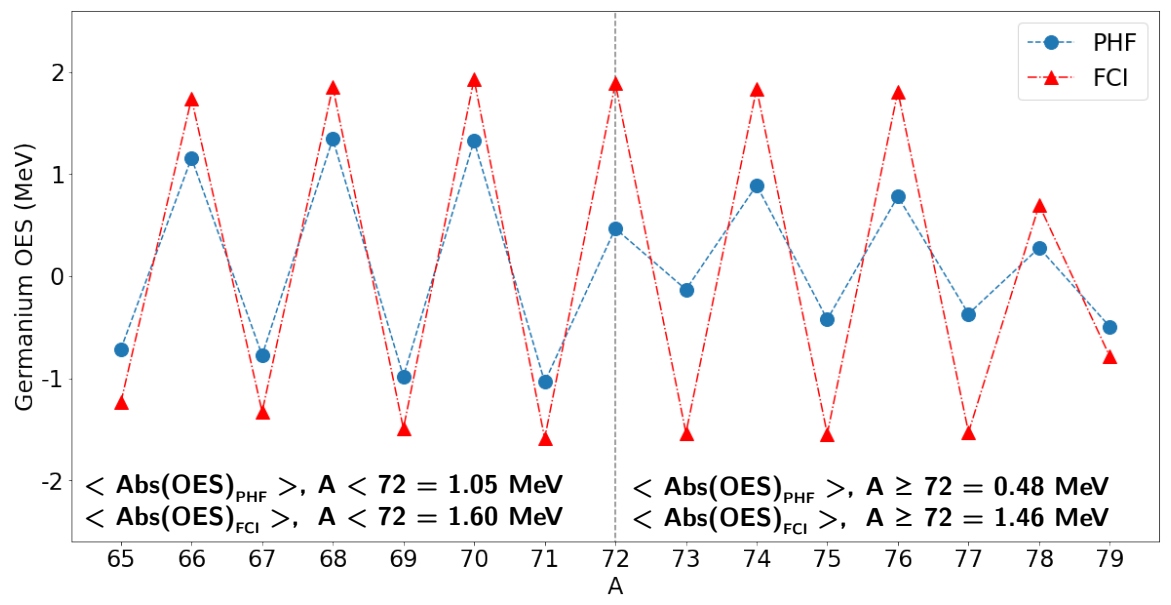

Figure 10. Odd-even staggering (OES) along the germanium isotopic chain. 


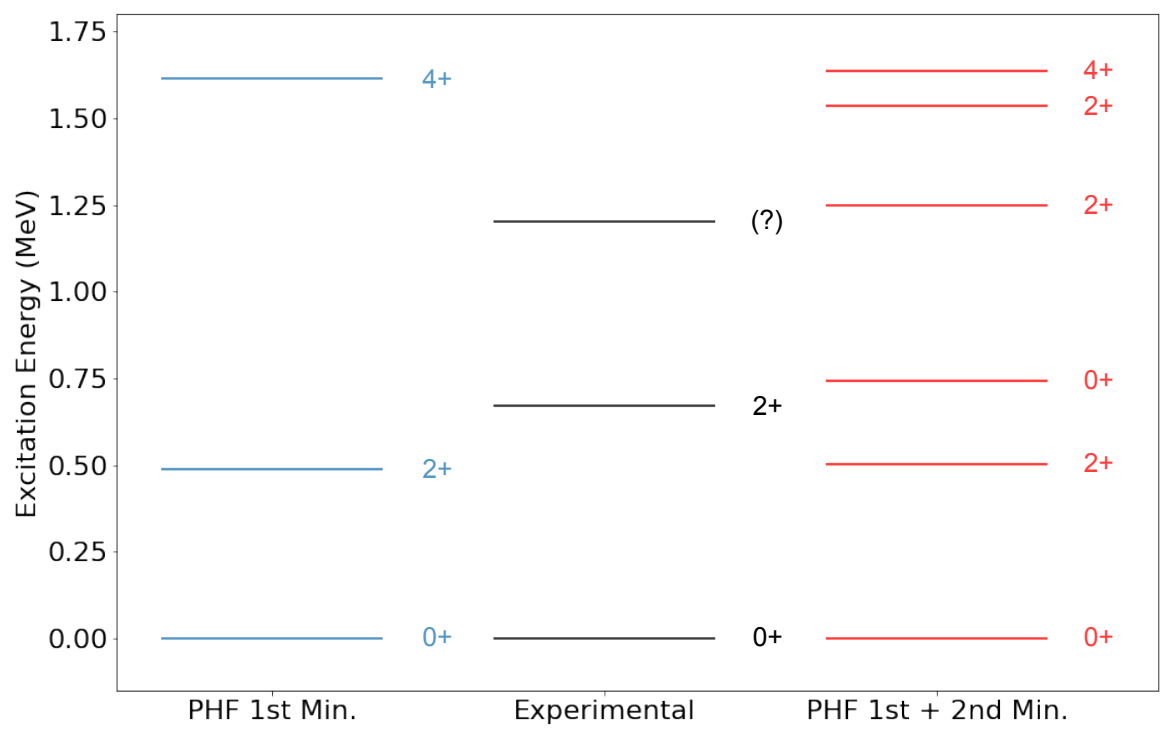

Figure 11. PHF calculation of ${ }^{40} \mathrm{Mg}$ compared to experimental results from Crawford et al. 17.

$0.7 \mathrm{MeV}$ for the $s d$ - and $p f$-shells, and around $0.4 \mathrm{MeV}$ for germanium isotopes).

An important concern is pairing. We have two contradictory pieces of information. The first is that for even-even nuclides we tend to get the $2_{1}^{+}$too low, or relatively speaking the ground state $0^{+}$is not low enough, and this trend increases as we move up in shells. Here the lack of treating pairing is a viable suspect. While one might expect pairing to also affect neutron separation energies and the odd-even staggering of binding energies, PHF provides a reasonable approximation to FCI calculations. When we increase the strength of pairing in chromium isotopes, however, the PHF estimate of the OES badly underperforms.

While methods to compute the overlap of Bogoliubov vacua have gotten more efficient [49, [50, it is nonetheless time-consuming to project out states of good particle number as well as good angular momentum. Given that the simpler PHF already encompasses many features of low-lying nuclear spectra, it is reasonable to ask whether simple configuration mixing of a few states, in the direction of generator coordinate methods or the Monte Carlo shell model, could compete with projected HFB. This question we leave to future work. We also find complex germanium spectra a challenge to reproduce, even when using shape coexistence. Thus germanium provides a stringent test of any beyond-mean-field method.

This material is based upon work supported by the U.S. Department of Energy, Office of Science, Office of Nuclear Physics, under Award Number DE-FG0203ER41272, and by the Office of High Energy Physics, under Award No. DESC0019465.

[1] Peter Ring and Peter Schuck. The nuclear many-body problem. Springer Science \& Business Media, 2004.

[2] P.J. Brussard and P.W.M. Glaudemans. Shell-model applications in nuclear spectroscopy. North-Holland Publishing Company, Amsterdam, 1977.

[3] Ian Stuart Towner. A shell model description of light nuclei. Clarendon Press, Oxford, 1977. 
[4] RD Lawson and RD Lawson. Theory of the nuclear shell model. Clarendon Press Oxford, 1980.

[5] B. A. Brown and B. H. Wildenthal. Status of the nuclear shell model. Annual Review of Nuclear and Particle Science, 38:29-66, 1988.

[6] E. Caurier, G. Martinez-Pinedo, F. Nowacki, A. Poves, and A. P. Zuker. The shell model as a unified view of nuclear structure. Reviews of Modern Physics, 77:427-488, 2005.

[7] Calvin W. Johnson, W. Erich Ormand, and Plamen G. Krastev. Factorization in large-scale many-body calculations. Computer Physics Communications, 184(12):2761 - 2774, 2013.

[8] Steven C Pieper and Robert B Wiringa. Quantum Monte Carlo calculations of light nuclei. Annual Review of Nuclear and Particle Science, 51(1):53-90, 2001.

[9] Gaute Hagen, Thomas Papenbrock, M Hjorth-Jensen, and David J Dean. Coupled-cluster computations of atomic nuclei. Reports on Progress in Physics, 77(9):096302, 2014.

[10] P-G Reinhard and K Goeke. The generator coordinate method and quantised collective motion in nuclear systems. Reports on Progress in Physics, 50(1):1, 1987.

[11] LM Robledo, TR Rodríguez, and RR Rodríguez-Guzmán. Mean field and beyond description of nuclear structure with the Gogny force: a review. Journal of Physics G: Nuclear and Particle Physics, 46(1):013001, 2018.

[12] P Klüpfel, J Erler, P-G Reinhard, and JA Maruhn. Systematics of collective correlation energies from self-consistent mean-field calculations. The European Physical Journal A, 37(3):343$355,2008$.

[13] Michio Honma, Takahiro Mizusaki, and Takaharu Otsuka. Nuclear shell model by the quantum Monte Carlo diagonalization method. Phys. Rev. Lett., 77:3315-3318, Oct 1996.

[14] T Otsuka, M Honma, T Mizusaki, N Shimizu, and Y Utsuno. Monte Carlo shell model for atomic nuclei. Progress in Particle and Nuclear Physics, 47(1):319-400, 2001.

[15] Carlos A Jiménez-Hoyos, Thomas M Henderson, Takashi Tsuchimochi, and Gustavo E Scuseria. Projected Hartree-Fock theory. The Journal of chemical physics, 136(16):164109, 2012.

[16] Kris Heyde and John L. Wood. Shape coexistence in atomic nuclei. Rev. Mod. Phys., 83:14671521, Nov 2011.

[17] H. L. Crawford, P. Fallon, A. O. Macchiavelli, P. Doornenbal, N. Aoi, F. Browne, C. M. Campbell, S. Chen, R. M. Clark, M. L. Cortés, M. Cromaz, E. Ideguchi, M. D. Jones, R. Kanungo, M. MacCormick, S. Momiyama, I. Murray, M. Niikura, S. Paschalis, M. Petri, H. Sakurai, M. Salathe, P. Schrock, D. Steppenbeck, S. Takeuchi, Y. K. Tanaka, R. Taniuchi, $\mathrm{H}$. Wang, and K. Wimmer. First spectroscopy of the near drip-line nucleus ${ }^{40} \mathrm{Mg}$. Phys. Rev. Lett., 122:052501, Feb 2019.

[18] B. Alex Brown and W. A. Richter. New "USD" Hamiltonians for the sd shell. Phys. Rev. C, 74:034315, Sep 2006.

[19] M. Honma, T. Otsuka, B. A. Brown, and T. Mizusaki. Effective interaction for $p f$-shell nuclei. Phys. Rev. C, 65:061301, May 2002.

[20] M. Honma, T. Otsuka, B. A. Brown, and T. Mizusaki. New effective interaction for $p f$-shell nuclei and its implications for the stability of the $N=Z=28$ closed core. Phys. Rev. C, 69:034335, Mar 2004.

[21] M Honma, T Otsuka, BA Brown, and T Mizusaki. Shell-model description of neutron-rich pf-shell nuclei with a new effective interaction GXPF1. Eur. Phys. J. A, 25(1):499-502, 2005.

[22] M. Honma, T. Otsuka, T. Mizusaki, and M. Hjorth-Jensen. New effective interaction for $f_{5} p g_{9^{-}}$ shell nuclei. Phys. Rev. C, 80:064323, Dec 2009.

[23] Yutaka Utsuno, Takaharu Otsuka, B. Alex Brown, Michio Honma, Takahiro Mizusaki, and Noritaka Shimizu. Shape transitions in exotic si and s isotopes and tensor-force-driven JahnTeller effect. Phys. Rev. C, 86:051301, Nov 2012.

[24] Calvin W Johnson, W Erich Ormand, Kenneth S McElvain, and Hongzhang Shan. Bigstick: A flexible configuration-interaction shell-model code. arXiv preprint arXiv:1801.08432, 2018.

[25] R. R. Whitehead, A. Watt, B. J. Cole, and I. Morrison. Computational methods for shell model calculations. Advances in Nuclear Physics, 9:123-176, 1977.

[26] I. Stetcu and C. W. Johnson. Random phase approximation vs exact shell-model correlation energies. Phys. Rev. C, 66:034301, 2002.

[27] Nicolas Schunck, Jacek Dobaczewski, W Satuła, P Bkaczyk, J Dudek, Yuan Gao, M Konieczka, K Sato, Yue Shi, XB Wang, et al. Solution of the Skyrme-Hartree-Fock-Bogolyubov equations in the Cartesian deformed harmonic-oscillator basis.(viii) hfodd (v2. 73y): A new version of the program. Computer Physics Communications, 216:145-174, 2017.

[28] J. A. White, S. E. Koonin, and D. J. Dean. Shell model Monte Carlo investigation of rare earth nuclei. Phys. Rev. C, 61:034303, Feb 2000.

[29] N. Schunck, J. Dobaczewski, J. McDonnell, J. Moré, W. Nazarewicz, J. Sarich, and M. V. 
Stoitsov. One-quasiparticle states in the nuclear energy density functional theory. Phys. Rev. C, 81:024316, Feb 2010.

[30] Sara Perez-Martin and L. M. Robledo. Microscopic justification of the equal filling approximation. Phys. Rev. C, 78:014304, Jul 2008.

[31] L. M. Robledo and G. F. Bertsch. Application of the gradient method to Hartree-FockBogoliubov theory. Phys. Rev. C, 84:014312, Jul 2011.

[32] Ionel Stetcu and Calvin W. Johnson. Gamow-Teller transitions and deformation in the protonneutron random phase approximation. Phys. Rev. C, 69:024311, Feb 2004.

[33] C. W. Johnson and K. D. O'Mara. Projection of angular momentum via linear algebra. Phys. Rev. C, 96:064304, 2017.

[34] C. W. Johnson and C. F. Jiao. Convergence and efficiency of angular momentum projection. J. Phys. G: Nucl. Part. Phys., 46:015101, 2019.

[35] M. R. Gunye and Chindhu S. Warke. Projected Hartree-Fock spectra of $2 s-1 d$-shell nuclei. Phys. Rev., 156:1087-1093, Apr 1967.

[36] RR Whitehead and A Watt. Exact shell-model and projected Hartree-Fock calculations for ${ }^{24} \mathrm{Mg}$. Physics Letters B, 35(3):189-190, 1971.

[37] RR Whitehead and A Watt. Shell-model and projected Hartree-Fock calculations for ${ }^{24} \mathrm{Mg}$, ${ }^{28} \mathrm{Si}$ and ${ }^{32} \mathrm{~S}$. Physics Letters B, 41(1):7-10, 1972.

[38] E Hammaren, KW Schmid, and Amand Faessler. Unrestricted symmetry-projected HartreeFock-Bogoliubov calculations for $s d$-shell nuclei. The European Physical Journal A-Hadrons and Nuclei, 2(4):371-377, 1998.

[39] R. Lecomte, G. Kajrys, S. Landsberger, P. Paradis, and S. Monaro. Shape coexistence and shape transitions in the even- $A$ Ge nuclei. Phys. Rev. C, 25:2812-2814, May 1982.

[40] Lu Guo, J. A. Maruhn, and P.-G. Reinhard. Triaxiality and shape coexistence in germanium isotopes. Phys. Rev. C, 76:034317, Sep 2007.

[41] C. W. Johnson, G. F. Bertsch, D. J. Dean, and I. Talmi. Generalized seniority from random Hamiltonians. Phys. Rev. C, 61:014311, Dec 1999.

[42] A. F. Lisetskiy, B. A. Brown, M. Horoi, and H. Grawe. New $T=1$ effective interactions for the $f_{5}-p_{3}-p_{1}-g_{9}$ model space: Implications for valence-mirror symmetry and seniority isomers. Phys. Rev. C, 70:044314, Oct 2004 .

[43] D. Verney, F. Ibrahim, C. Bourgeois, S. Essabaa, S. Galès, L. Gaudefroy, D. Guillemaud-Mueller, F. Hammache, C. Lau, F. Le Blanc, A. C. Mueller, O. Perru, F. Pougheon, B. Roussière, J. Sauvage, and O. Sorlin. Low-energy states of ${ }_{31}^{81} \mathrm{Ga}_{50}$ : Proton structure of the nuclei close to ${ }^{78} \mathrm{Ni}$. Phys. Rev. C, 76:054312, Nov 2007.

[44] E Chabanat, P Bonche, P Haensel, J Meyer, and R Schaeffer. A Skyrme parametrization from subnuclear to neutron star densities. Nuclear Physics A, 627(4):710-746, 1997.

[45] M. Bender, K. Rutz, P.-G. Reinhard, J. A. Maruhn, and W. Greiner. Shell structure of superheavy nuclei in self-consistent mean-field models. Phys. Rev. C, 60:034304, Aug 1999.

[46] W. Satuła, J. Dobaczewski, and W. Nazarewicz. Odd-even staggering of nuclear masses: Pairing or shape effect? Phys. Rev. Lett., 81:3599-3602, Oct 1998.

[47] G. F. Bertsch, C. A. Bertulani, W. Nazarewicz, N. Schunck, and M. V. Stoitsov. Odd-even mass differences from self-consistent mean field theory. Phys. Rev. C, 79:034306, Mar 2009.

[48] H. L. Crawford, P. Fallon, A. O. Macchiavelli, R. M. Clark, B. A. Brown, J. A. Tostevin, D. Bazin, N. Aoi, P. Doornenbal, M. Matsushita, H. Scheit, D. Steppenbeck, S. Takeuchi, H. Baba, C. M. Campbell, M. Cromaz, E. Ideguchi, N. Kobayashi, Y. Kondo, G. Lee, I. Y. Lee, J. Lee, K. Li, S. Michimasa, T. Motobayashi, T. Nakamura, S. Ota, S. Paschalis, M. Petri, T. Sako, H. Sakurai, S. Shimoura, M. Takechi, Y. Togano, H. Wang, and K. Yoneda. Shell and shape evolution at $N=28$ : The ${ }^{40} \mathrm{Mg}$ ground state. Phys. Rev. C, 89:041303, Apr 2014.

[49] G. F. Bertsch and L. M. Robledo. Symmetry restoration in Hartree-Fock-Bogoliubov based theories. Phys. Rev. Lett., 108:042505, Jan 2012.

[50] BG Carlsson and J Rotureau. A new and practical formulation for overlaps of Bogoliubov vacua. arXiv preprint arXiv:2010.08459, 2020. 